\title{
Conceptual study for a sub-pupil instrument having 4 high order adaptive optics paths for parallel multi-wavelength high contrast imaging, and medium resolution spectrometry
}

\author{
Frederic Y. J. Gonte*a, Pierre. Bourget ${ }^{\mathrm{a}}$, Julien Girard ${ }^{\mathrm{a}}$, Pierre Haguenauer ${ }^{\mathrm{a}}$, Dimitri Mawet ${ }^{\mathrm{a}}$ \\ ${ }^{a}$ European Southern Observatory, Alonso de Cordova 3107, Santiago, Vitacura 19001, Chile
}

\begin{abstract}
We present the concept of an instrument that will create 4 circular sub-pupils of $3 \mathrm{~m}$ in diameter. Each sub-pupil path will be corrected by a high order adaptive optics system (SR $80 \%$ in $\mathrm{H})$ without spider and M2 obstruction. These four independent channels, obviously all pointed towards the same field, allows the possibility of covering totally different parts of the electromagnetic spectrum simultaneously without compromising Signal to Noise Ratio. Each channel can be dedicated to very specialized but complementary purposes: high contrast imaging, pseudo-wide field imaging, high precision multi-color photometry, medium-resolution spectroscopy, polarimetry and sparse-aperture masking
\end{abstract}

Keywords: high contrast imaging, coronagraphy, adaptive optics, exoplanet,

\section{INTRODUCTION}

The concept is based on an experience achieved at Palomar where the AO system of the telescope was used on a $1.5 \mathrm{~m}$ unobscured sub-pupil to obtain a high Strehl ratio (>90\% at $\mathrm{H}$ and $\mathrm{Ks}$ ) and a pupil without obscuration to achieve a very high contrast using a four quadrant phase mask coronagraph [1][2][3].

We propose here a concept to be applied on the VLT, but it could be exported to any large telescope, based on the last technologies developed in adaptive optics, coronagraphy and cryogenic detectors.

\section{SCIENCE CASES}

The science drivers for this concept are directly linked to the unique ability to reach the highest contrasts at the smallest angular separation in diffraction elements (thanks to the absence of central obscuration). Also the four parallel feed can be exploited towards high contrast color-color discrimination of very low mass objects. Spectral Energy Distributions (SEDs) with four points from 1 to $5 \mu \mathrm{m}$ can constraint very well stellar spectral types and age models and are often missing because instrument like 2-MASS, IRAS or SPITZER cannot resolve spatially the components of multiple stellar systems.

We identified the following (and no-exhaustive) science drivers:

1 - very low mass stars and exoplanet search and spectral characterization.

Inspired by the Lyot Project [11], SP can reach the same goals and add the possibility of performing consistent multiwavelengths surveys that can be complementary to the SPHERE surveys (i.e. NIRSUR). This can be done with state of the art coronagraphs whether it will be focal plane phase masks (i.e the annular groove phase mask) or pupil plane apodizers (i.e apodizing phase plate).

2- ultra cool brown dwarfs (Y/T type):

if used in a slower lower order mode, the adaptive optics can provide very good sensitivity to image multiple brown dwarfs binaries down to the limit of the Initial Mass Function and we can even imagine detect free floating Jupiter-mass objects in the nearby molecular clouds and characterize them spectro-photometrically thanks to the use of line to continuum filters ( $\mathrm{CH} 4$ and $\mathrm{H}$ for example) at glance and simultaneously.

Ground-based and Airborne Instrumentation for Astronomy IV, edited by lan S. McLean, Suzanne K. Ramsay, Hideki Takami, Proc. of SPIE Vol. 8446, 84467Z · @ 2012 SPIE · CCC code: 0277-786X/12/\$18 - doi: 10.1117/12.927162 
3- Differential Polarimetry to image debris disks and push down the detection limits for extra-solar planets (like for SPHERE/ZIMPOL but at IR-wavelengths). Indeed we could operate in pupil tracking mode and have each of the symmetric arms of this instrument see a different polarization two by two without the need of having a Wollaston prism but rather a rotation quarter-wave plate in two of the arms. That could be done simultaneously in $\mathrm{J}$ and $\mathrm{H}$ bands. Of course each component or coating's polarization properties would have to be well characterized. By using pupil tracking one can make sure the telescope M3 and the common-path de-rotator crossed and synchronized, stabilizing the pupil and the instrumental polarization (the Nasmyth instrument thus behaves like a Cassegrain instrument). This is currently been investigated with NACO.

\section{OPTOMECHANICAL CONCEPT}

\subsection{Warm part}

The instrument is installed at the Nasmyth platform of the telescope. An optical derotator containing a fast steering mirror to stabilize the pupil on the 4 deformable mirror is positioned at the focus of the telescope. An off-axis parabola images the pupil on the 4 deformable mirrors. The four paths are then separated and two off axis parabola reimage each sub-pupil for the SHS (Shack-Hartmann Sensor) of the AO system. A dichroic is used in each path to separate the visible light used by the SHS from the infrared light used for science. On the scientific path the pupil is imaged on the fast steering mirror of the derotator to stabilize the field on the mask of the coronagraph and eliminate all environmental effects like vibrations. The 4 coronagraphs are contained in two cryostats. The main warm bench I sonly compos of mirrors (except the dichroics) all silver coated and producing no chromaticity. The 4 optical paths are exactly symmetrical and have reduced differential polarization effects..

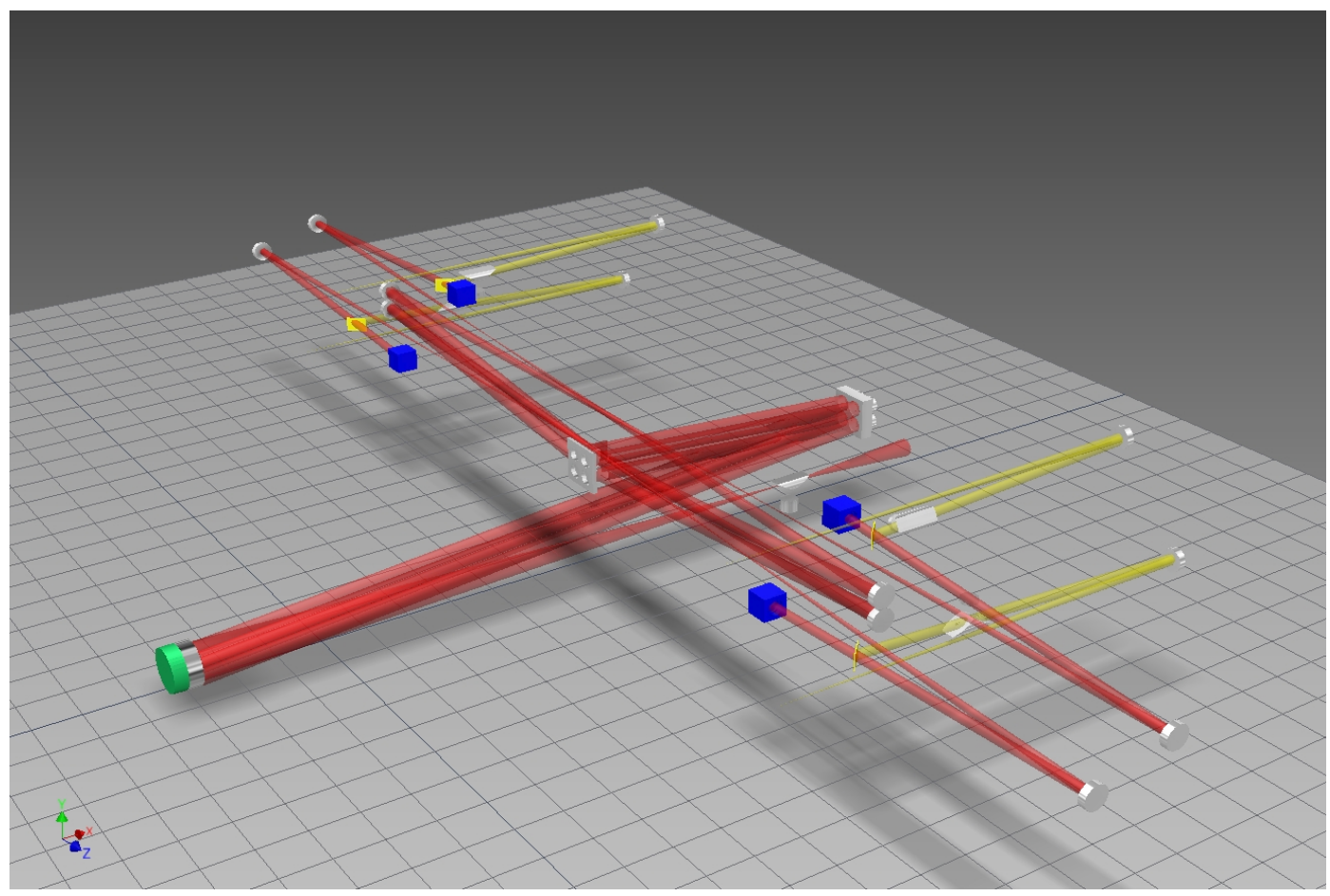

Figure 1: Opto-mechanical concept of the warm part.

\subsection{Cold part}

The four coronagraphs are contained in two cryostats. Each coronagraph is composed of an image planed where the vortex mask is positioned and a pupil plane for Lyot stop and grism. HAWAII 2RG [6] are used as detectors. The cryostats cooling system is based on PTC to limit global vibrations. 


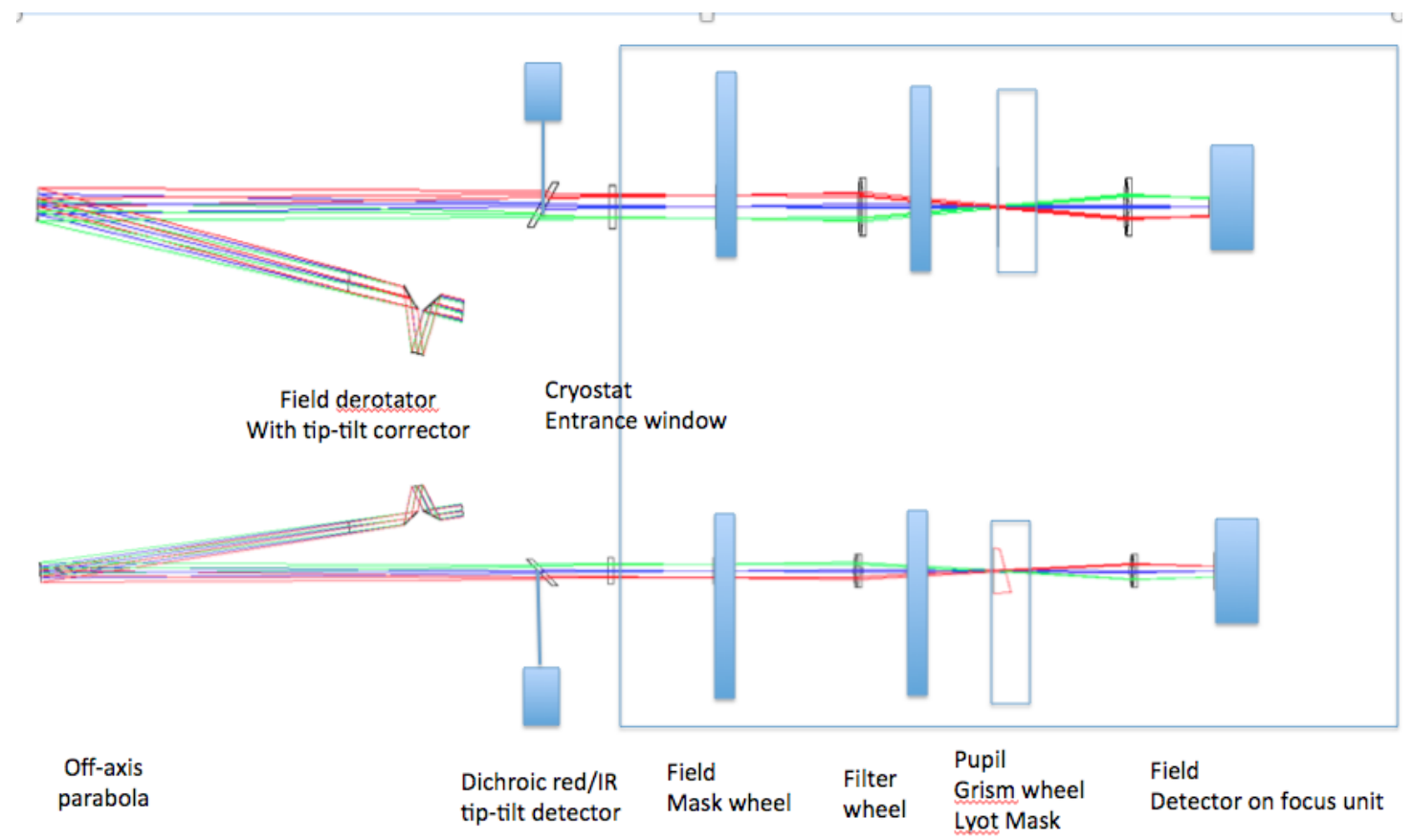

Figure 2: Opto-mechanical concept of the cold part. The mask, the grism and the detectors are contained in the cryostat

\section{ADAPTIVE OPTICS SYSTEM}

Each optical path has its own adaptive optics system working independently from the others. It means that the instruments will contains four complete adaptive optics systems based on membrane mirror, a high rate Shack Hartman wavefront sensor and a real time computer having a latency inferior to $1 \mathrm{~ms}$.

\subsection{Deformable mirror}

Deformable mirror such as the one developed at ALPAO [7] are commercially available and can be easily adapted to this instrument. They have already been tested at ESO and are currently being used on the PEACE experiment.

Clear Aperture size

Number of actuators

Distance between two actuators

Geometry of actuators

Mirror surface quality in closed loop

Wavefront tip/tilt stroke

Wavefront astigmatism and defocus

Wavefront inter-actuator stroke

Non linearity errors

Settling time

$24.5 \mathrm{~mm}$
277
$1.5 \mathrm{~mm}$
Square grid
Error $<7.0 \mathrm{~nm}$ RMS
$>+/-60 \mu \mathrm{m}$
$>+/-45 \mu \mathrm{m}$
$>3.0 \mu \mathrm{m}$
$<3 \%$
$1 \mathrm{~ms}$

Table 1. Specification for the DM 

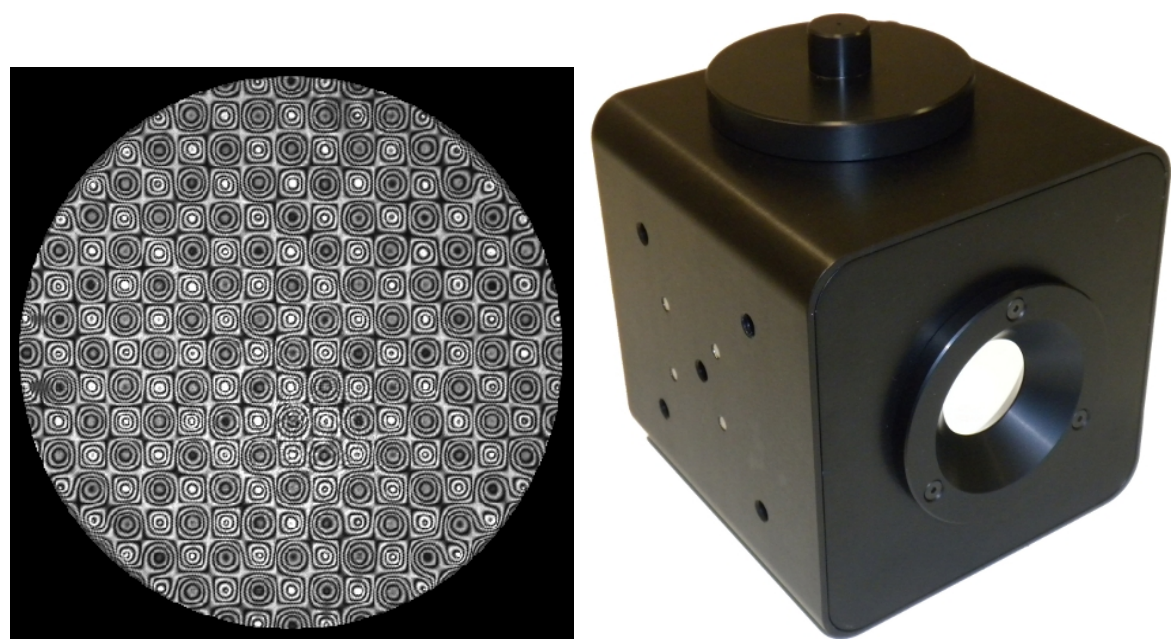

Figure 3: Opto-mechanical concept of the cold part.

\subsection{Wavefront sensor}

ESO has developed during the last years a new CCD dedicated to wavefront sensor for adaptive optics for the instrument SPHERE [9] and the Adaptive Optics Facility [10]. The performances are:

- RON:

$1.0 \mathrm{e}-/ \mathrm{pix} /$ frame

- Dark: $1.2 \mathrm{e}-/ \mathrm{pix} / \mathrm{s}$

- Excess noise:

2

- Charge diffusion: FWHM 0.55 pix

- Frequrency:

$$
1.4 \mathrm{KHz}
$$

\subsection{Real Time Computer}

The real time computer can be based on the one designed for the adaptive optics system to be installed on the Auxiliary telescope of the VLT called NAOMI or even the one designed for the Infrared Shack-Hartmann Sensor designed for Gravity. These RTCs can have latency below $1 \mathrm{~ms}$. Another solution is to choose one commercially available as such the one developed by the company ALPAO.

\section{ADAPTIVE OPTICS SIMULATIONS}

Simulations using the POALA packages [4] have been performed to verify the potential performances of a simple AO system when used on 3-m sub-pupils. The conditions taken are a median seeing of 0 ". 85 with an outer scale of $25 \mathrm{~m}$ and based on 9 turbulent layers of atmosphere. It is observed that we obtain a Strehl ratio higher than 0.8 in $\mathrm{J}$ band up to magnitude 8 and in $\mathrm{H}, \mathrm{K}$, and $\mathrm{L}$ up to magnitude 10. Three cases with different number of electrodes and loop speed have been simulated. It shows that it is better to improve the measurement speed that the number of lenslet. We propose then to work with a $16 * 16$ actuators mirror at a maximum frequency of $1 \mathrm{KHz}$. 


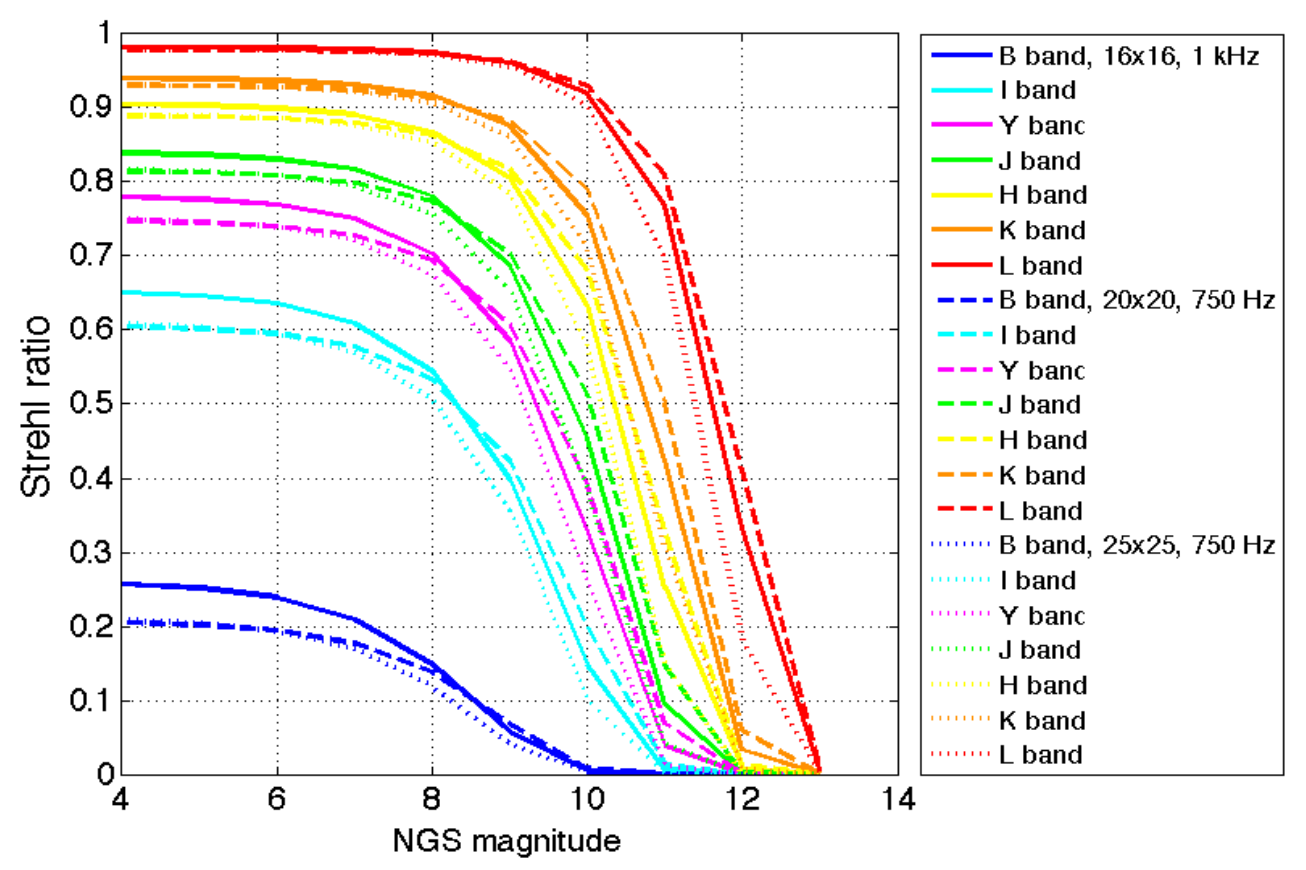

Figure 4: AO performances simulations

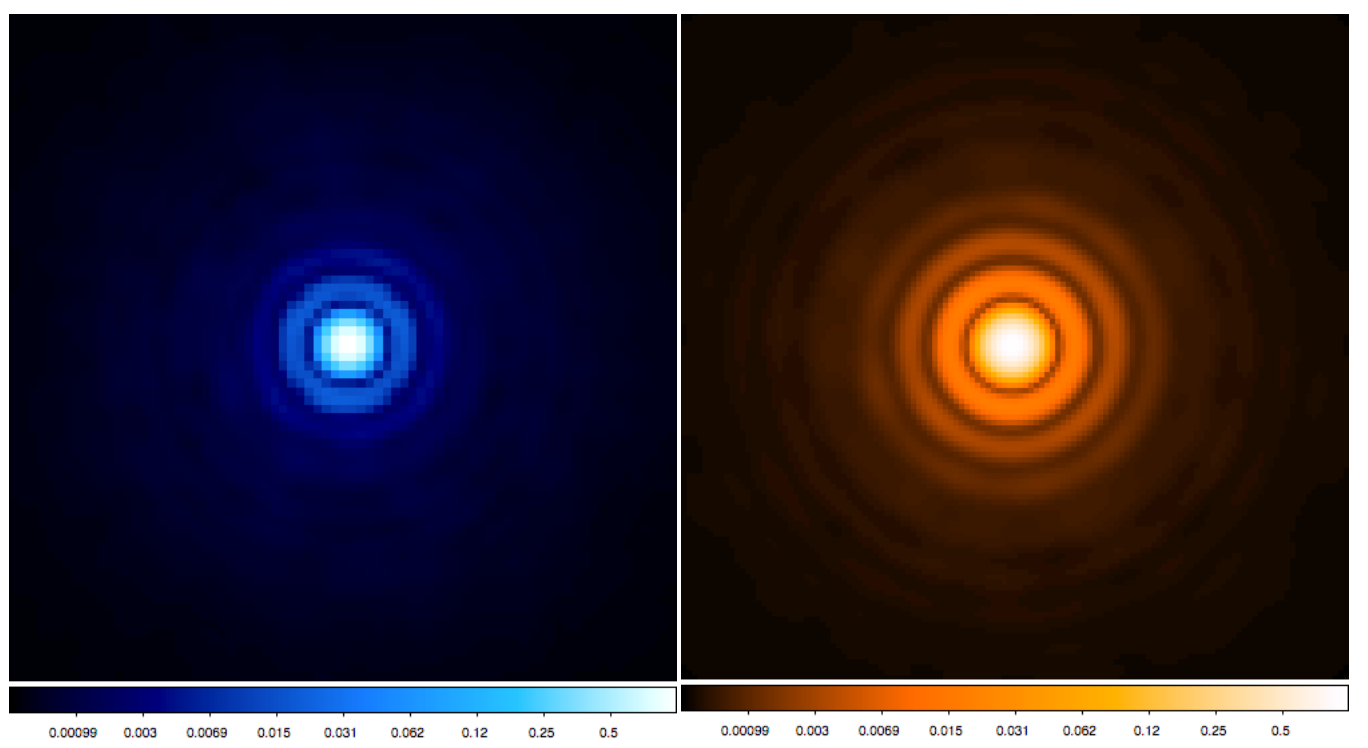

Figure 5: PSF obtained from the simulation ( $\mathrm{H}$ band on the left and $\mathrm{L}$ band on the right) 


\section{CORONAGRAPH TECHNOLOGY}

The field mask proposed for the four coronagraphs is of the Vortex type. It has demonstrated excellent result in laboratory recently [8].

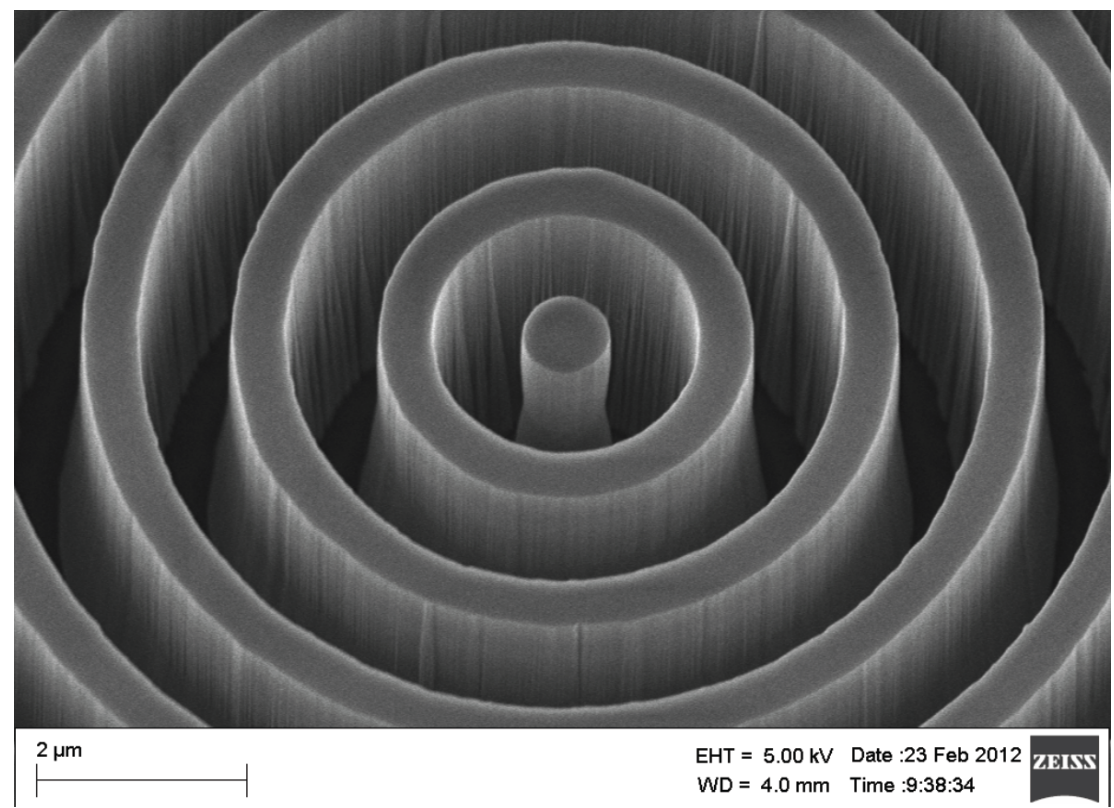

Figure 6: photo of the center of the vortex mask.

The spectrograph imager integrated to the coronagraph is based on grism to obtain low to medium spectrum resolution in the resolution range of 500 to 1500 in the 5 bandpass of interest $(\mathrm{J}, \mathrm{H}, \mathrm{K}, \mathrm{L}, \mathrm{M})$. Each coronagraph will be equipped with a Hawaii 2RG detector [6] from Teledyne controlled by NGC recently developed at ESO

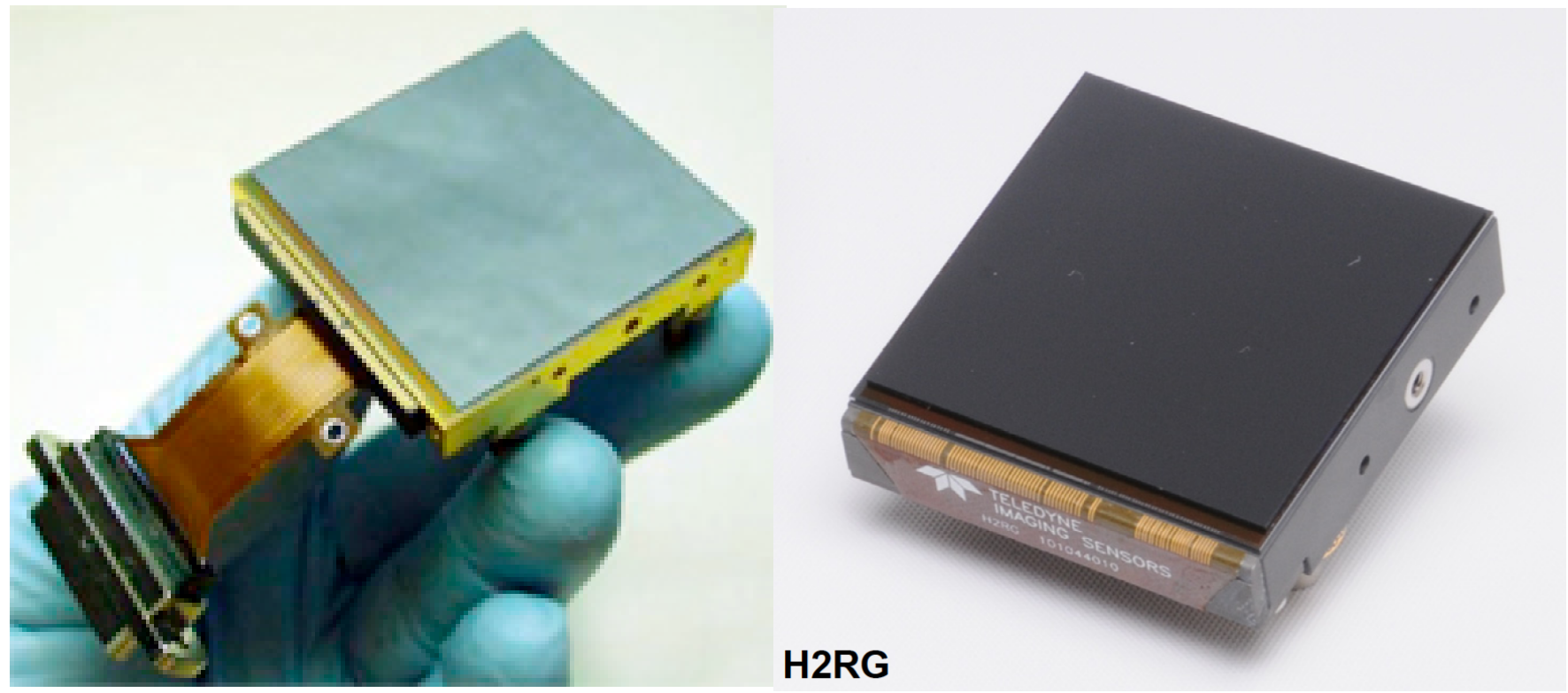

Figure 7: two photos of the Hawaii 2RG developed by Teledyne 


\section{CORONAGRAPH SIMULATION}

Simulation have been made base on the CAOS software package [5]. The atmospheric conditions are the same as for the $\mathrm{AO}$ simulations the other input are given below:

- telescope diam $3 \mathrm{~m}$

- obscuration diam $0 \mathrm{~m}$

- $16 x 16$ subapertures

- $1 \mathrm{kHz}$ WFS

- delay $2 \mathrm{~ms}$

- $\quad$ gain 0.3

- WFS mean wavelength 0.65 micron

- readout noise $1 \mathrm{e}$ -

- dark current $1.2 \mathrm{e}-$

- $\quad$ instrument jitter 3 mas

- $\quad$ static NCPA $20 \mathrm{~nm}$

- AO calibration $4 \mathrm{~nm}$

- Fresnel propagation $5 \mathrm{~nm}$

- beam shit $8 \mathrm{~nm}$

- defocus $3 \mathrm{~nm}$

- pupil shear $1 \%$

- $\quad$ pupil rotation $1 \mathrm{deg}$

- differential rotation $9 \mathrm{~nm}$

- differential beam shift $3 \mathrm{~nm}$
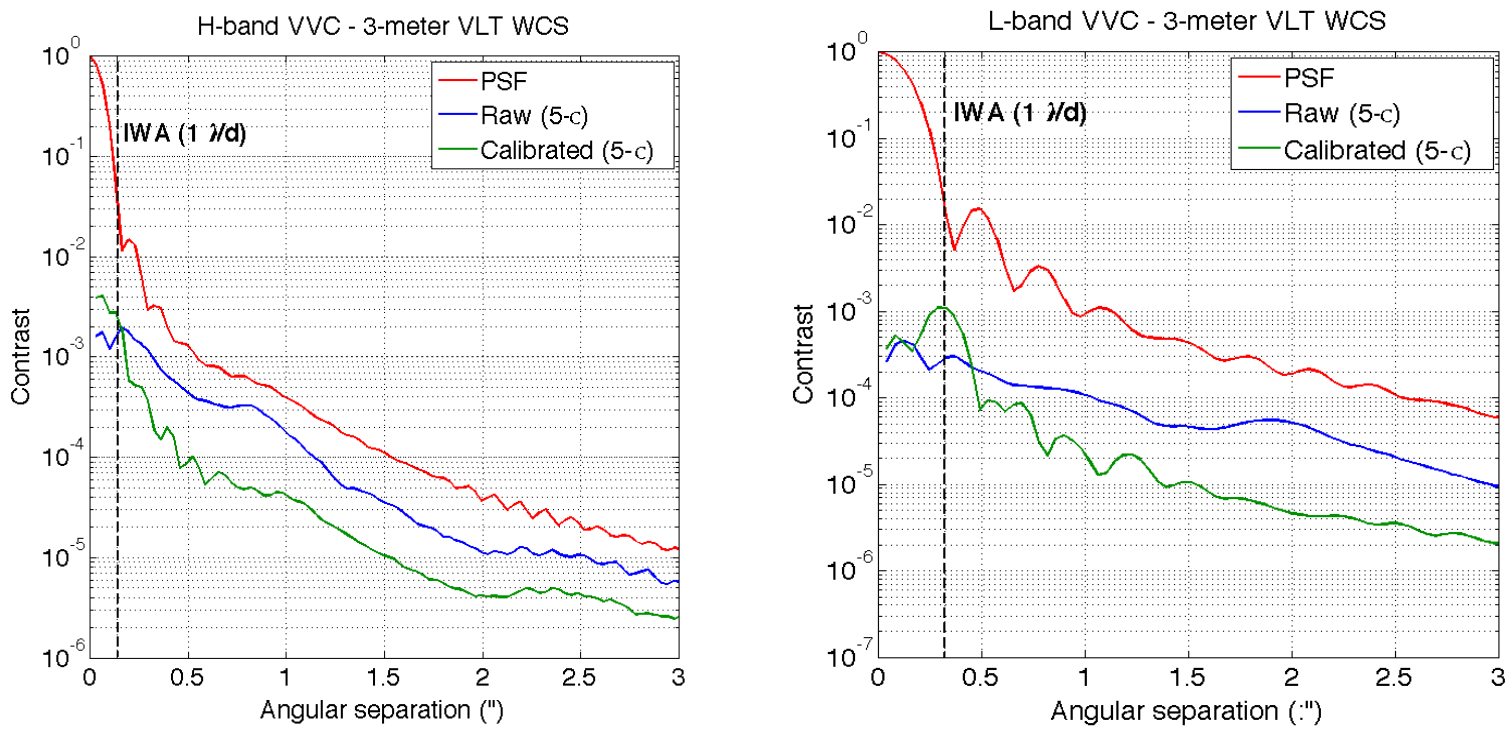

Figure 8: Coronagraph performances in $\mathrm{H}$ and $\mathrm{L}$ band 


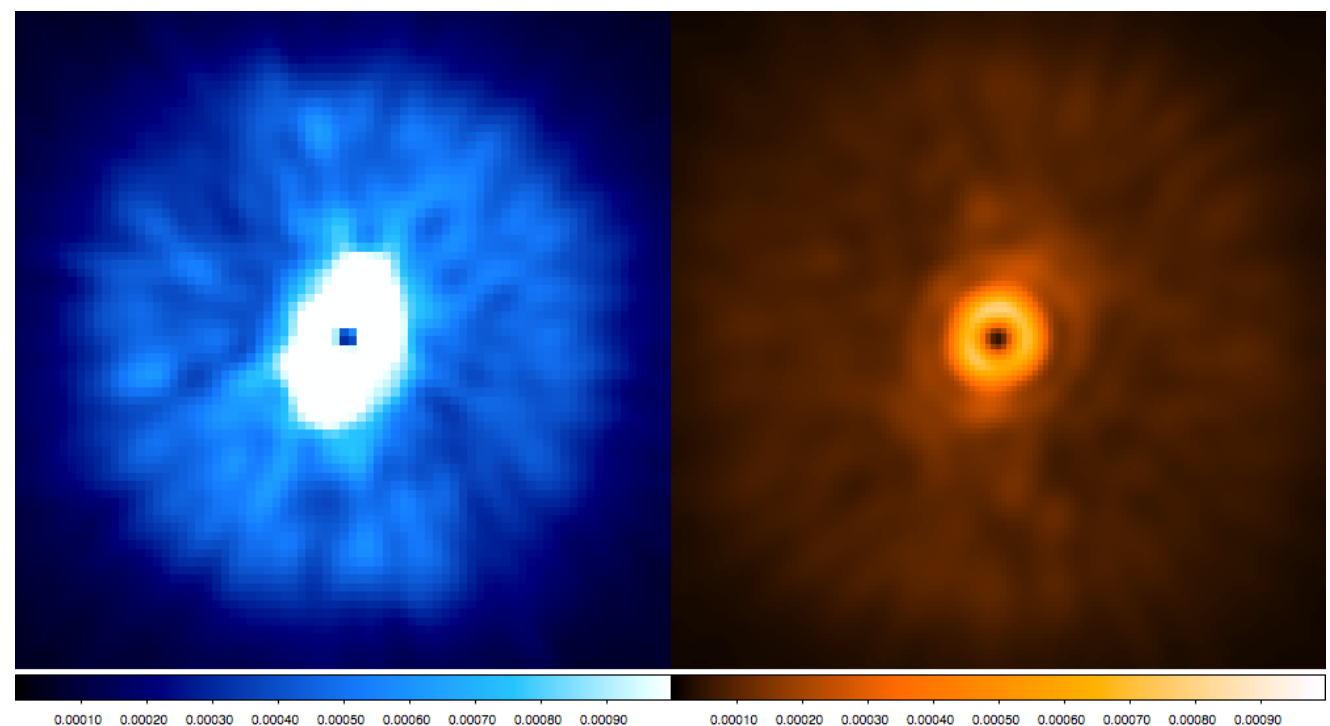

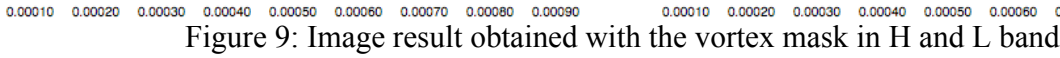

\section{CONCLUSION}

The concept shows that a window on high contrast imaging and spectrometry can be opened with parallel measurement at different wavelengths. It is possible to make the imaging and the spectrograph at the same time of the star and its environment. The contrast is increased compare to a full pupil with spider.

The full instrument is based on existing and demonstrated technologies. The components are highly repetitive and no systems require long development as they are all on the market. The instrument can be contained in an envelop of 4 millions Euros and 50 man/year in a time line smaller than 5 years

\section{REFERENCES}

[1] Serabyn, E. et al, Extreme adaptive optics imaging with a clear and well corrected off-axis subaperture, The Astrophysical Journal, 658:1386 Y 1391, 2007 April 1

[2] Haguenauer, P. et al, An off-axis Four-Quadrant Phase-Mask coronagraph for Palomar: high contrast near bright stars imager, Proc. of SPIE Vol. 5905 59050S-2

[3] Serabyn, E. et al, An off-axis Four-Quadrant Phase-Mask : concept and first results, Proc. of SPIE Vol. 5905 59050S-2

[4] Jollisaint, L, Synthetic Modeling of Astronomical Closed Loop Adaptive Optics, Journal of the European Optical Society, 5, 2010.

[5] Carbillet, M. et al. Modelling astronomical adaptive optics: I. The Software Package CAOS, MNRAS 356 (4), 1253 (2005).

[6] http://www.teledyne-si.com/imaging/hawaii2rg.html

[7] www.alpao.fr

[8] Mawet, D. et al. Vector Vortex Coronagraph technology developments for space-based and ground-based telescopes: status update, and recent test results, spirit of Lyot 2010

[9] Petit, C. The SPHERE XAO system SAXO: integration test and laboratory final performances Proc. Of SPIE [844771]

[10] Arsenault, R. et al. The ESO adaptive optics facility integration completed and readiness for system test Proc. Of SPIE [8447-19]

[11] Oppenheimer, B. R. et al. "The Lyot Project: Toward Exoplanet Images and Spectra," Terrestrial Planet Finder Technical Conference and Exposition, C. Lindensmith, ed. (NASA/JPL Publications; March 2004). 